Pacific

Journal of

Mathematics

\title{
KÄHLER METRICS ON SINGULAR TORIC VARIETIES
}

Dan Burns, Victor Guillemin and Eugene LeRMan 


\title{
KÄHLER METRICS ON SINGULAR TORIC VARIETIES
}

\author{
DAN BURNS, Victor GUILLEMIN AND EUGENE LERMAN
}

\begin{abstract}
We extend Guillemin's formula for Kähler potentials on toric manifolds to singular quotients of $\mathbb{C}^{N}$ and $\mathbb{C} \boldsymbol{P}^{N}$.
\end{abstract}

\section{Introduction}

Let $G$ be a torus with Lie algebra $\mathfrak{g}$ and integral lattice $\mathbb{Z}_{G} \subset \mathfrak{g}$. Let $u_{1}, \ldots, u_{N} \in \mathbb{Z}_{G}$ be a set of primitive vectors which span $\mathfrak{g}$ over $\mathbb{R}$. Let $\lambda_{1}, \ldots, \lambda_{N} \in \mathbb{R}$ and let

$$
P=P_{u, \lambda}:=\left\{\eta \in \mathfrak{g}^{*} \mid\left\langle\eta, u_{j}\right\rangle-\lambda_{j} \geq 0,1 \leq j \leq N\right\}
$$

be the corresponding polyhedral set. We assume that $P$ has a nonempty interior and that the collection of inequalities defining $P$ is minimal: if we drop the condition that $\left\langle\eta, u_{j}\right\rangle-\lambda_{j} \geq 0$ for some index $j$ then the resulting set is strictly bigger than $P$.

A well-known construction of Delzant, suitably tweaked, produces a symplectic stratified space $M_{P}$ with an effective Hamiltonian action of the torus $G$ and associated moment map $\phi=\phi_{P}: M_{P} \rightarrow \mathfrak{g}^{*}$ such that $\phi\left(M_{P}\right)=P$. We will review the construction below. The space $M_{P}$ is a symplectic quotient of $\mathbb{C}^{N}$ by a compact abelian subgroup $K$ of the standard torus $\mathbb{T}^{N}$. Therefore, by a theorem of Heinzner and Loose [1994] $M_{P}$ is a complex analytic space. Moreover $M_{P}$ is a Kähler space; see [Heinzner and Loose 1994, (3.5)] and [Heinzner et al. 1994]. Even though in general the space $M_{P}$ is singular, the preimages of open faces of $P$ under the moment map $\phi_{P}$ are smooth Kähler manifolds. The main results of the paper are formulas for the Kähler forms on these manifolds. In particular we will show that the Kähler form $\omega$ on the preimage $\phi_{P}^{-1}(\stackrel{\circ}{P})$ of the interior $\stackrel{\circ}{P}$ of the polyhedral set $P$ is given by

$$
\omega=\sqrt{-1} \partial \bar{\partial} \phi_{P}^{*}\left(\sum_{j=1}^{N} \lambda_{j} \log \left(u_{j}-\lambda_{j}\right)+u_{j}\right),
$$

MSC2000: primary 32J27; secondary 14M25, 53D20.

Keywords: Kähler metric, toric, Kähler potential, Kähler reduction, stratified space.

Supported in part by NSF grants DMS-0104047 and DMS-0514070 (DB), DMS-0104116 and DMS0408993 (VG), and DMS-0204448(EL). 
where we think of $u_{j} \in \mathbb{Z}_{G}$ as a function on $\mathfrak{g}^{*}$.

Formula (1-1) was originally proved by Guillemin in the case where $M_{P}$ is a compact manifold (and thus $P$ is a simple unimodular polytope, also known as a Delzant polytope). It was extended to the case of compact orbifolds by Abreu [2001]. Calderbank, David and Gauduchon gave two new proofs of Guillemin's formula (for orbifolds) in [Calderbank et al. 2003]. One of their proofs was simplified further in [Burns and Guillemin 2004].

As we just mentioned, for generic values of $\lambda$ the polyhedral set $P$ is simple and consequently $M_{P}$ is at worse an orbifold. But for arbitrary values of $\lambda$ it may have more serious singularities. Of particular interest is the singular case where $P$ is a cone on a simple polytope. Then there is only one singular point, and the link of the singularity is a Sasakian orbifold. Such orbifolds, especially the ones with Sasaki-Einstein metrics, have attracted some attention in string theory. They play a role in the AdS/CFT correspondence [Martelli and Sparks 2004].

If the polyhedral set $P$ is a polytope, that is, if $P$ is compact, then as a symplectic space $M_{P}$ may also be obtained as a symplectic quotient of $\mathbb{C} P^{N}$. In this case the Fubini-Study form on $\mathbb{C P}^{N}$ will induce a Kähler structure on $M_{P}$, which is different from the one induced by the flat metric on $\mathbb{C}^{N}$ even in the case where $M_{P}$ is smooth. We will give a formula for this Kähler structure as well.

The methods of this paper are quite close to that of [Calderbank et al. 2003]. In particular the key Lemma 3.3 is a direct corollary of Proposition 2 in that reference.

\section{The "Delzant" construction: toric varieties as Kähler quotients}

It will be convenient for us to fix the following notation. As in the introduction, let $G$ be a torus with Lie algebra $\mathfrak{g}$ and integral lattice $\mathbb{Z}_{G} \subset \mathfrak{g}$. Let $u_{1}, \ldots, u_{N} \in \mathbb{Z}_{G}$ be a set of primitive vectors which span $\mathfrak{g}$ over $\mathbb{R}$. Let $\lambda_{1}, \ldots, \lambda_{N} \in \mathbb{R}$ and let

$$
P=P_{u, \lambda}:=\left\{\eta \in \mathfrak{g}^{*} \mid\left\langle\eta, u_{j}\right\rangle-\lambda_{j} \geq 0, \quad 1 \leq j \leq N\right\}
$$

be the corresponding polyhedral set. As above we assume that $P$ has the nonempty interior and that the collection of inequalities defining $P$ is minimal. Let $A: \mathbb{Z}^{N} \rightarrow$ $\mathbb{Z}_{G}$ be the $\mathbb{Z}$-linear map given by

$$
A\left(x_{1}, \ldots, x_{N}\right)=\sum x_{i} u_{i} .
$$

That is, $A$ is defined by sending the standard basis vector $e_{i}$ of $\mathbb{Z}^{N}$ to $u_{i}$. Let $A$ also denote the $\mathbb{R}$-linear extension $\mathbb{R}^{N} \rightarrow \mathfrak{g}$. Let $\mathfrak{k}=\operatorname{ker} A$ and let $B: \mathfrak{k} \rightarrow \mathbb{R}^{N}$ denote the inclusion. The map $A$ induces a surjective map of Lie groups

$$
\bar{A}: \mathbb{T}^{N}=\mathbb{R}^{N} / \mathbb{Z}^{N} \rightarrow \mathfrak{g} / \mathbb{Z}_{G}=G .
$$


Let $K=\operatorname{ker} \bar{A}$ and let $\bar{B}: K \rightarrow \mathbb{T}^{N}$ denote the corresponding inclusion. The group $K$ is a compact abelian group which need not be connected. It's easy to see that the Lie algebra of $K$ is $\mathfrak{k}$.

We have a short exact sequence of abelian Lie algebras:

$$
0 \rightarrow \mathfrak{k} \stackrel{B}{\rightarrow} \mathbb{R}^{N} \stackrel{A}{\rightarrow} \mathfrak{g} \rightarrow 0 .
$$

Let

$$
0 \rightarrow \mathfrak{g}^{*} \stackrel{A^{*}}{\rightarrow}\left(\mathbb{R}^{N}\right)^{*} \stackrel{B^{*}}{\rightarrow} \mathfrak{k}^{*} \rightarrow 0
$$

be the dual sequence. Note that $\operatorname{ker} B^{*}=A^{*}\left(\mathfrak{g}^{*}\right)=\mathfrak{k}^{\circ}$ where $\mathfrak{k}^{\circ}$ denotes the annihilator of $\mathfrak{k}$ in $\left(\mathbb{R}^{N}\right)^{*}$. Let $\left\{e_{i}{ }^{*}\right\}$ denote the dual basis of $\left(\mathbb{R}^{N}\right)^{*}$ and let $\lambda=\sum \lambda_{i} e_{i}{ }^{*}$. We note that

$$
\left(B^{*}\right)^{-1}\left(B^{*}(-\lambda)\right)=-\lambda+\mathfrak{k}^{\circ}=-\lambda+A^{*}\left(\mathfrak{g}^{*}\right) .
$$

In particular $\left(B^{*}\right)^{-1}\left(B^{*}(-\lambda)\right)$ is the image of the affine embedding

$$
\iota_{\lambda}: \mathfrak{g}^{*} \hookrightarrow\left(\mathbb{R}^{N}\right)^{*}, \quad \iota_{\lambda}(\ell)=-\lambda+A^{*}(\ell) .
$$

Lemma 2.1. Let $P$ be the polyhedral set defined by (2-1) above.

(1) There exists a Kähler space $M_{P}$ with an effective holomorphic Hamiltonian action of the torus $G$ so that the image of the associated moment map $\phi_{P}$ : $M_{P} \rightarrow \mathfrak{g}^{*}$ is $P$.

(2) For every open face $\stackrel{\circ}{F}$, the preimage $\phi_{P}^{-1}(\stackrel{\circ}{F})$ is the Kähler quotient of a complex torus $\left(\mathbb{C}^{\times}\right)^{N_{F}}$ by a compact subgroup $K_{F}$ of the compact torus $\mathbb{T}^{N_{F}} \subset$ $\left(\mathbb{C}^{\times}\right)^{N_{F}}$. Here the number $N_{F}$ and the group $K_{F}$ depend on the face $F$.

(3) If the set $P$ is bounded, then $M_{P}$ can also be constructed as a Kähler quotient of $\mathbb{C P}^{N}$.

Proof. For every index $i$ and any $\eta \in \mathfrak{g}^{*}$

$$
\left\langle\eta, A e_{i}\right\rangle-\lambda_{i}=\left\langle A^{*} \eta, e_{i}\right\rangle-\left\langle\sum \lambda_{j} e_{j}{ }^{*}, e_{i}\right\rangle=\left\langle A^{*} \eta-\lambda, e_{i}\right\rangle=\left\langle\iota_{\lambda}(\eta), e_{i}\right\rangle .
$$

Therefore

$$
\iota_{\lambda}(P)=\left\{\ell \in\left(\mathbb{R}^{N}\right)^{*} \mid\left\langle\ell, e_{i}\right\rangle \geq 0,1 \leq i \leq N\right\} \cap \iota_{\lambda}\left(\mathfrak{g}^{*}\right) .
$$

More generally, if $\stackrel{\circ}{F} \subset P$ is an open face, there is a unique subset $I_{F}=I \subset$ $\{1, \ldots, N\}$ so that

$$
\stackrel{\circ}{F}=\bigcap_{j \notin I}\left\{\eta \in \mathfrak{g}^{*} \mid\left\langle\eta, u_{j}\right\rangle-\lambda_{j}>0\right\} \cap \bigcap_{j \in I}\left\{\eta \in \mathfrak{g}^{*} \mid\left\langle\eta, u_{j}\right\rangle-\lambda_{j}=0\right\} .
$$

Therefore

$$
\iota_{\lambda}(\stackrel{\circ}{F})=\iota_{\lambda}\left(\mathfrak{g}^{*}\right) \cap \bigcap_{j \notin I}\left\{\ell \in\left(\mathbb{R}^{N}\right)^{*} \mid\left\langle\ell, e_{j}\right\rangle>0\right\} \cap \bigcap_{j \in I}\left\{\ell \in\left(\mathbb{R}^{N}\right)^{*} \mid\left\langle\ell, e_{j}\right\rangle=0\right\} .
$$


The moment map $\phi$ for the action of $\mathbb{T}^{N}$ on $\left(\mathbb{C}^{N}, \sqrt{-1} \sum d z_{j} \wedge d \bar{z}_{j}\right)$ is given by

$$
\phi(z)=\sum\left|z_{j}\right|^{2} e_{j}^{*}
$$

Hence

$$
\phi\left(\mathbb{C}^{N}\right)=\left\{\ell \in\left(\mathbb{R}^{N}\right)^{*} \mid\left\langle\ell, e_{i}\right\rangle \geq 0, \quad 1 \leq i \leq N\right\} .
$$

The moment map $\phi_{K}$ for the action of $K$ on $\mathbb{C}^{N}$ is the composition

$$
\phi_{K}=B^{*} \circ \phi \text {. }
$$

Let $v=B^{*}(-\lambda)$. We argue that

$$
\phi\left(\phi_{K}^{-1}(v)\right)=\iota_{\lambda}(P) .
$$

Indeed,

$$
\begin{aligned}
\phi_{K}^{-1}(v) & =\phi^{-1}\left(\left(B^{*}\right)^{-1}(v)\right)=\phi^{-1}\left(\left(B^{*}\right)^{-1}\left(B^{*}(-\lambda)\right)\right) \\
& =\phi^{-1}\left(\iota_{\lambda}\left(\mathfrak{g}^{*}\right)\right)=\phi^{-1}\left(\phi\left(\mathbb{C}^{N}\right) \cap \iota_{\lambda}\left(\mathfrak{g}^{*}\right)\right)=\phi^{-1}\left(\iota_{\lambda}(P)\right) .
\end{aligned}
$$

Therefore

$$
\phi\left(\phi_{K}^{-1}(v)\right)=\iota_{\lambda}(P)
$$

The restriction

$$
\left.\phi\right|_{\phi_{K}^{-1}(v)}
$$

descends to a map

$$
\bar{\phi}: M_{P} \equiv \phi_{K}^{-1}(v) / K \rightarrow \iota_{\lambda}\left(\mathfrak{g}^{*}\right) .
$$

It is not hard to see that the composition $\phi_{P}$ of $\bar{\phi}$ with the isomorphism

$$
\iota_{\lambda}\left(\mathfrak{g}^{*}\right) \stackrel{\simeq}{\rightarrow} \mathfrak{g}^{*}
$$

is a moment map for the action of $G$ on the symplectic quotient (symplectic stratified space) $M_{P}$. Since the isomorphism $\iota_{\lambda}\left(\mathfrak{g}^{*}\right) \rightarrow \mathfrak{g}^{*}$ obviously maps $\iota_{\lambda}(P)$ to $P$, we conclude that the image of $\phi_{P}: M_{P} \rightarrow \mathfrak{g}^{*}$ is exactly $P$. This proves (1).

To prove (2) we define a bit more notation. For a subset $I \subset\{1, \ldots, N\}$ we define the corresponding coordinate subspace

$$
V_{I}:=\left\{z \in \mathbb{C}^{N} \mid j \in I \Rightarrow z_{j}=0\right\} .
$$

Its "interior" $\stackrel{\circ}{V}_{I}$ is defined by

$$
\stackrel{\circ}{V}_{I}:=\left\{z \in \mathbb{C}^{N} \mid j \in I \Leftrightarrow z_{j}=0\right\} .
$$

Also, let

$$
\mathbb{T}_{I}^{N}:=\left\{a \in \mathbb{T}^{N} \mid j \notin I \Rightarrow a_{j}=1\right\} .
$$

The sets $V_{I}, \stackrel{\circ}{V}_{I}$ are Kähler submanifolds of $\mathbb{C}^{N}$ preserved by the action of $\mathbb{T}^{N}$. Both are fixed by $\mathbb{T}_{I}^{N}$, with $\stackrel{\circ}{V}_{I}$ being precisely the set of points of orbit type $\mathbb{T}_{I}^{N}$. 
The restriction $\left.\phi_{K}\right|_{V_{I}}$ is a moment map for the action of $K$ on $\stackrel{\circ}{V_{I}}$. Moreover, for any $v \in \mathfrak{k}^{*}$

$$
\phi_{K}^{-1}(v) \cap \stackrel{\circ}{V}_{I}=\left(\left.\phi_{K}\right|_{V_{I}}\right)^{-1}(v) .
$$

Hence

$$
\left(\phi_{K}^{-1}(v) \cap \stackrel{\circ}{V}_{I}\right) / K=\left(\left.\phi_{K}\right|_{V_{I}}\right)^{-1}(v) / K .
$$

While the action of $K$ on $\stackrel{\circ}{V}_{I}$ need not be free, the action of

$$
K_{I}:=K /\left(K \cap \mathbb{\mathbb { T }}_{I}^{N}\right)
$$

on $\stackrel{\circ}{V}_{I}$ is free. Therefore, the quotient $\left(\phi_{K}^{-1}(v) \cap \stackrel{\circ}{V}_{I}\right) / K$ may be interpreted as a regular Kähler quotient of $\stackrel{\circ}{V}_{I}$ by the Hamiltonian action of $K_{I}$ :

$$
\left(\phi_{K}^{-1}(v) \cap \stackrel{\circ}{V}_{I}\right) / K=\stackrel{\circ}{V}_{I} / / \nu_{I} K_{I}
$$

for an appropriate value $v_{I} \in \mathfrak{k}_{I}^{*}$ of the $K_{I}$ moment map.

Given a face $F$, let $I=I_{F}$ be the corresponding subset of $\{1, \ldots, N\}$. By (2-3),

$$
\begin{aligned}
\left\{z \in \mathbb{C}^{N} \mid \phi(z) \in \iota_{\lambda}(\stackrel{\circ}{F})\right\} & =\left\{z \in \mathbb{C}^{N} \mid \phi(z) \in \iota_{\lambda}\left(\mathfrak{g}^{*}\right),\left\langle\phi(z), e_{j}\right\rangle\left\{\begin{array}{l}
>0 \text { for } j \notin I, \\
=0 \text { for } j \in I
\end{array}\right\}\right. \\
& =\phi_{K}^{-1}(v) \cap \stackrel{\circ}{V}_{I} .
\end{aligned}
$$

Therefore,

$$
\phi_{K}^{-1}(v) \cap \stackrel{\circ}{V}_{I}=\phi^{-1}\left(\iota_{\lambda}(\stackrel{\circ}{F})\right) .
$$

It follows from the definition of $\phi_{P}$ that

$$
\left(\phi_{K}^{-1}(v) \cap \stackrel{\circ}{V}_{I}\right) / K=\phi_{P}^{-1}(\stackrel{\circ}{F}) .
$$

By (2-4) we conclude that

$$
\phi_{P}^{-1}(\stackrel{\circ}{F})=\stackrel{\circ}{V}_{I} / / v_{I} K_{I}
$$

This proves (2).

If $P$ is compact, then $\iota_{\lambda}(P) \subset\left(\mathbb{R}^{N}\right)^{*}$ is bounded. Hence $\iota_{\lambda}(P)$ is contained in a sufficiently large multiple of the standard simplex. Any such simplex is the image of $\mathbb{C} \mathrm{P}^{N}$ under the moment map for the standard action of $\mathbb{T}^{N}$ with the Kähler form on $\mathbb{C P}^{N}$ being the appropriate multiple of the standard Fubini-Study form. This proves (3).

Remark 2.2. It follows from the results of Heinzner and his collaborators (email communication), in particular of Heinzner and Huckleberry [1996], that the action of $G$ on $M_{P}$ extends to an action the complexified group $G^{\mathbb{C}}$. This action of $G^{\mathbb{C}}$ has a dense open orbit. In other words, $M_{P}$ is a toric Kähler space. 


\section{Kähler potentials, Legendre transforms and symplectic quotients}

As we mentioned in the introduction, the line of argument of this section is quite close to the approach in [Calderbank et al. 2003], and Lemma 3.3 can be easily deduced form Proposition 2 of that reference. We keep our exposition self-contained.

We start by recalling a result of Guillemin [1994, Theorems 4.2, 4.3]:

Lemma 3.1. Suppose the action of $\mathbb{T}^{N}$ on $\left(\mathbb{C}^{\times}\right)^{N}=\mathbb{R}^{N} \times \sqrt{-1} \mathbb{t}^{N}$ preserves a Kähler form $\omega$ and is Hamiltonian. Then there exists a $\mathbb{T}^{N}$-invariant function $f$ on $\left(\mathbb{C}^{\times}\right)^{N}$ such that $\omega=i \partial \bar{\partial} f$. Additionally

$$
\mathscr{L}_{f} \circ \pi:\left(\mathbb{C}^{\times}\right)^{N} \rightarrow\left(\mathbb{R}^{N}\right)^{*}
$$

is a moment map for the action of $\mathbb{T}^{N}$ on $\left(\left(\mathbb{C}^{\times}\right)^{N}, \omega\right)$. Here $\pi: \mathbb{R}^{N} \times \sqrt{-1} \mathbb{T}^{N} \rightarrow$ $\mathbb{R}^{N}$ is the projection and $\mathscr{L}_{f}: \mathbb{R}^{N} \rightarrow\left(\mathbb{R}^{N}\right)^{*}$ is the Legendre transform of $f$, where we have identified $f \in C^{\infty}\left(\left(\mathbb{C}^{\times}\right)^{N}\right)^{\mathbb{T}^{N}}$ with a function on $\mathbb{R}^{N}$.

The same result holds with $\left(\mathbb{C}^{\times}\right)^{N}$ replaced by $U \times \sqrt{-1} \mathbb{T}^{N}$ for any contractible open set $U \subset \mathbb{R}^{N}$.

Lemma 3.2. Let $f: V \rightarrow \mathbb{R}$ be a (strictly) convex function on a finite dimensional vector space $V$, let $A: W \rightarrow V$ be an injective linear map, $x \in V$ be a point and

$$
j: W \rightarrow V, \quad j(w)=A w+x
$$

an affine map. Then $f \circ j: W \rightarrow \mathbb{R}$ is (strictly) convex and the associated Legendre transform $\mathscr{L}_{f \circ j}: W \rightarrow W^{*}$ is given by

$$
\mathscr{L}_{f \circ j}=A^{*} \circ \mathscr{L}_{f} \circ j
$$

where $A^{*}: V^{*} \rightarrow W^{*}$ is the dual map.

Proof. By the chain rule and the definition of the Legendre transform,

$$
\mathscr{L}_{f \circ j}(w)=d(f \circ j)_{w}=d f_{j(w)} \circ d j_{w}=\mathscr{L}_{f}(j(w)) \circ A=A^{*} \circ \mathscr{L}_{f} \circ j(w)
$$

for any $w \in W$.

Lemma 3.3. Let $f \in C^{\infty}\left(\mathbb{R}^{N}\right)$ be a strictly convex function and $\omega=\sqrt{-1} \partial \bar{\partial} \pi_{N}^{*} f$ the corresponding $\mathbb{T}^{N}$-invariant Kähler form on $\left(\mathbb{C}^{\times}\right)^{N}=\mathbb{R}^{N} \times \sqrt{-1} \mathbb{T}^{N}$ (here $\pi_{N}:\left(\mathbb{C}^{\times}\right)^{N} \rightarrow \mathbb{R}^{N}$ is the projection $)$. Let $\phi=\mathscr{L}_{f} \circ \pi_{N}:\left(\mathbb{C}^{\times}\right)^{N} \rightarrow\left(\mathbb{R}^{N}\right)^{*}$ denote the associated moment map.

Let $K \subset \mathbb{T}^{N}$ be a closed subgroup and let $G=\mathbb{T}^{N} / K$. For any $v \in \mathfrak{k}^{*}$ the symplectic quotient

$$
\left(\mathbb{C}^{\times}\right)^{N} / /{ }_{v} K
$$

is biholomorphic to $U \times \sqrt{-1} G \subset \mathfrak{g} \times \sqrt{-1} G=G^{\mathbb{C}}$ where $U \subset \mathfrak{g}$ is an open contractible set. Hence the reduced Kähler form $\omega_{\nu}$ has a potential $f_{v}$. 
Moreover, the Legendre-Fenchel dual $f_{v}^{*}$ of the Kähler potential $f_{v}$ is given by

$$
f_{v}^{*}=f^{*} \circ \iota_{\lambda}
$$

where $\iota_{\lambda}: \mathfrak{g}^{*} \rightarrow\left(\mathbb{R}^{N}\right)^{*}$ is the affine embedding (2-2) and $-\lambda$ is a point in $\left(B^{*}\right)^{-1}(v)$. Proof. It is no loss of generality to assume that the group $K$ is connected. Then $\mathbb{T}^{N} \simeq K \times G$. Consequently $\mathbb{R}^{N} \simeq \mathfrak{k} \times \mathfrak{g}$ and the short exact sequence

$$
0 \rightarrow \mathfrak{k} \stackrel{B}{\rightarrow} \mathbb{R}^{N} \stackrel{A}{\rightarrow} \mathfrak{g} \rightarrow 0
$$

splits. Let

$$
\pi_{K}: \mathbb{R}^{N} \rightarrow \mathfrak{k} \quad \text { and } \quad \iota_{\mathfrak{g}}: \mathfrak{g} \rightarrow \mathbb{R}^{N}
$$

denote the maps defined by the splitting. The moment map $\phi_{K}:\left(\mathbb{C}^{\times}\right)^{N} \rightarrow \mathfrak{k}^{*}$ for the action of $K$ on $\left(\left(\mathbb{C}^{\times}\right)^{N}, \omega\right)$ is the composition

$$
\phi_{K}=B^{*} \circ \phi=B^{*} \circ \mathscr{L}_{f} \circ \pi_{N} .
$$

Let

$$
\Delta=\left(B^{*}\right)^{-1}(v) \cap \phi\left(\left(\mathbb{C}^{\times}\right)^{N}\right)=\left(B^{*}\right)^{-1}(v) \cap \mathscr{L}_{f}\left(\mathbb{R}^{N}\right) .
$$

Then $\Delta$ is the intersection of an affine hyperplane with a convex set, hence is contractible.

Since the action of $K$ on $\phi_{K}^{-1}(v)$ is free, $K^{\mathbb{C}} \cdot \phi_{K}^{-1}(v)$ is an open subset of $\left(\mathbb{C}^{\times}\right)^{N}$ and $K^{\mathbb{C}}$ acts freely on it. Moreover, for each $x \in \phi_{K}^{-1}(v)$ the orbit $K^{\mathbb{C}} \cdot x$ intersects the level set $\phi_{K}^{-1}(v)$ transversely and

$$
K^{\mathbb{C}} \cdot x \cap \phi_{K}^{-1}(v)=K \cdot x
$$

(see [Guillemin and Sternberg 1982, pp. 526-527]). It follows that the restriction

$$
\left.\pi_{K}\right|_{\mathscr{L}_{f}^{-1}(\Delta)}: \mathscr{L}_{f}^{-1}(\Delta) \rightarrow \mathfrak{k}
$$

is $1-1$ and a local diffeomorphism. Hence

$$
U=\pi_{K}\left(\mathscr{L}_{f}^{-1}(\Delta)\right)
$$

is a contractible open set.

On the other hand, the restriction $\left.\omega\right|_{\phi_{K}^{-1}(v)}$ descends to a Kähler form $\omega_{v}$ on the symplectic quotient

$$
\left.\left(\mathbb{C}^{\times}\right)^{N}\right) / /{ }_{\nu} K:=\phi_{K}^{-1}(v) / K
$$

Moreover, since $\omega$ is $\mathbb{T}^{N}$ invariant, $\omega_{\nu}$ is $G$-invariant. Note that

$$
\left.\left(\mathbb{C}^{\times}\right)^{N}\right) / /{ }_{\nu} K \simeq U \times \sqrt{-1} G \subset G^{\mathbb{C}} .
$$

By Lemma 3.1 there exists $f_{v} \in C^{\infty}(U)$ such that

$$
\omega_{v}=\sqrt{-1} \partial \bar{\partial} f_{\nu} .
$$


The potential $f_{v}$ defines a moment map

$$
\phi_{G}: U \times \sqrt{-1} G \rightarrow \mathfrak{g}^{*}
$$

with

$$
\phi_{G}=\mathscr{L}_{f_{v}} \circ \pi_{G},
$$

where $\pi_{G}: U \times \sqrt{-1} G \rightarrow U$ is the projection. Moreover, by adjusting $f_{v}$ [Burns and Guillemin 2004] we may arrange for the diagram

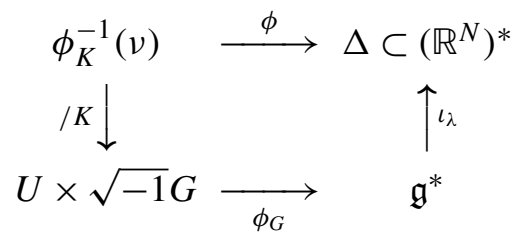

to commute. That is, the moment map $\phi_{G}$ is defined up to a constant and the potential $f_{v}$ is defined up to a pluriharmonic $G$-invariant function. By adding an appropriate pluriharmonic function to $f_{v}$ we can change $\phi_{G}$ by any constant we want. Since $\phi=\mathscr{L}_{f} \circ \pi_{N}$ and since $\phi_{G}=\mathscr{L}_{f_{v}} \circ \pi_{G}$, it follows from (3-2) that the diagram

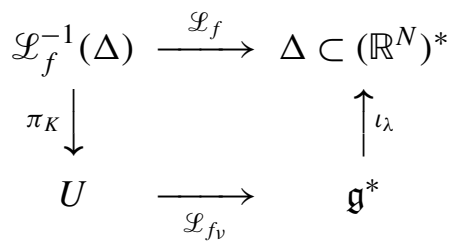

commutes as well. Since $\left(\mathscr{L}_{f_{v}}\right)^{-1}=\mathscr{L}_{f_{v}^{*}}$, where $f_{v}^{*}$ is the Legendre-Fenchel dual of $f_{v}$,

$$
\mathscr{L}_{f_{v}^{*}}=\pi_{K} \circ\left(\mathscr{L}_{f}\right)^{-1} \circ \iota_{\lambda}=\pi_{K} \circ\left(\mathscr{L}_{f^{*}}\right) \circ \iota_{\lambda} .
$$

By Lemma 3.2,

$$
\mathscr{L}_{f_{v}^{*}}=\mathscr{L}_{f^{*} \circ \iota_{\lambda}}
$$

Therefore, up to a constant, $f_{v}^{*}=f^{*} \circ \iota_{\lambda}$.

\section{From potentials to dual potentials and back again}

We start by making two observations. Let $V$ be a real finite dimensional vector space, $V^{*}$ its dual, $\mathcal{O} \subset V$ an open set, $\varphi \in C^{\infty}(\mathcal{O})$ a strictly convex function, $\mathscr{L}_{\varphi}$ : $\mathcal{O} \rightarrow V^{*}$ the Legendre transform (which we assume to be invertible), $0^{*}=\mathscr{L}_{\varphi}(\mathbb{O})$ and $\varphi^{*} \in C^{\infty}\left(0^{*}\right)$ the Fenchel dual of $\varphi$.

Lemma 4.1. Under these assumptions, $\varphi=\left(\mathscr{L}_{\varphi}\right)^{*} h$, where $h: 0^{*} \rightarrow \mathbb{R}$ is given by

$$
h(\eta)=\left\langle\eta,\left(d \varphi^{*}\right)_{\eta}\right\rangle-\varphi^{*}(\eta)
$$

where we think of $\left(d \varphi^{*}\right)_{\eta} \in T_{\eta}^{*} O^{*}$ as an element of $\left(V^{*}\right)^{*}=V$. 
Proof. By the definition of the Fenchel dual, $\varphi(s)+\varphi^{*}(\eta)=\langle\eta, s\rangle$ for $\eta=\mathscr{L}_{\varphi}(s)$. Hence

$$
\varphi(s)=\langle\eta, s\rangle-\varphi^{*}(\eta)=\left\langle\eta,\left(\mathscr{L}_{\varphi}\right)^{-1}(\eta)\right\rangle-\varphi^{*}(\eta)=\left\langle\eta, \mathscr{L}_{\varphi^{*}}(\eta)\right\rangle-\varphi^{*}(\eta)
$$

and the result follows since $\mathscr{L}_{\varphi^{*}}(\eta)=\left(d \varphi^{*}\right)_{\eta}$.

Lemma 4.2. We keep the above notation. Suppose additionally that the dual potential $\varphi^{*}$ has the special form

$$
\varphi^{*}(\eta)=\sum_{i=1}^{N} f_{i}\left(u_{i}(\eta)-\lambda_{i}\right),
$$

where $u_{1}, \ldots, u_{N}$ are vectors in $V$ (thought of as linear functionals $u_{i}: V^{*} \rightarrow \mathbb{R}$ ), $\lambda_{i} \in \mathbb{R}$ are constants and $f_{i}$ 's are functions of one variable. Then

$$
h(\eta)=\sum_{i=1}^{N}\left(f_{i}^{\prime}\left(u_{i}(\eta)-\lambda_{i}\right) u_{i}(\eta)-f_{i}\left(u_{i}(\eta)-\lambda_{i}\right)\right) .
$$

Proof. Observe that

$$
d\left(f_{i} \circ\left(u_{i}-\lambda_{i}\right)\right)_{\eta}=f_{i}^{\prime}\left(u_{i}(\eta)-\lambda_{i}\right) d\left(u_{i}-\lambda_{i}\right)_{\eta}=f_{i}^{\prime}\left(u_{i}(\eta)-\lambda_{i}\right) u_{i}
$$

since $u_{i}$ is linear. Hence

$$
\left\langle\eta,\left(d \varphi^{*}\right)_{\eta}\right\rangle=\left\langle\eta, \sum f_{i}^{\prime}\left(u_{i}(\eta)-\lambda_{i}\right) u_{i}\right\rangle=\sum f_{i}^{\prime}\left(u_{i}(\eta)-\lambda_{i}\right) u_{i}(\eta)
$$

and (4-1) follows from Lemma 4.1.

Example 4.3. We use the lemma above to argue that for the standard action of $\mathbb{T}^{N}$ on $\left(\mathbb{C}^{N}, \sqrt{-1} \partial \bar{\partial}\|z\|^{2}\right)$, the dual potential $\varphi^{*}$ is given by

$$
\varphi^{*}=\sum_{i=1}^{N} e_{i} \log e_{i}
$$

where $\left\{e_{1}, \ldots, e_{N}\right\}$ is the standard basis of $\mathbb{R}^{N}=\operatorname{Lie}\left(\mathbb{T}^{N}\right)$.

Indeed, the homogeneous moment map $\Phi: \mathbb{C}^{N} \rightarrow\left(\mathbb{R}^{N}\right)^{*}$ for the standard action of $\mathbb{T}^{N}$ is given by

$$
\Phi(z)=\sum\left|z_{j}\right|^{2} e_{j}^{*},
$$

where $\left\{e_{j}^{*}\right\}$ is the basis dual to $\left\{e_{j}\right\}$. Hence

$$
\|z\|^{2}=\Phi^{*}\left(\sum e_{j}\right) .
$$

On the other hand, if $\varphi^{*}=\sum e_{j} \log e_{j}$, then

$$
\varphi^{*}=\sum f \circ e_{j}
$$


where $f(x)=x \log x$. Since $f^{\prime}(x)=\log x+1$, (4-1) becomes

$$
h=\sum\left(\log e_{j}+1\right) e_{j}-\sum e_{j} \log e_{j}=\sum e_{j} .
$$

Therefore, $\varphi^{*}=\sum e_{j} \log e_{j}$ is, indeed, the dual potential.

We are now in position to prove (1-1).

Theorem 4.4. Let $G$ be a torus, $P \subset \mathfrak{g}^{*}$ the polyhedral set defined by (2-1), $M_{P}=$ $\mathbb{C}^{N} / /{ }_{\nu} K$ the Kähler $G$-space with moment map $\phi_{P}: M_{P} \rightarrow \mathfrak{g}^{*}$ constructed in Lemma 2.1 (1). Then the Kähler form $\omega_{P}$ on $\stackrel{\circ}{M}_{P}:=\phi_{P}^{-1}(\stackrel{\circ}{P})$ is given by

$$
\omega_{P}=\sqrt{-1} \partial \bar{\partial} \phi_{P}^{*}\left(\sum_{j=1}^{N} \lambda_{j} \log \left(u_{j}-\lambda_{j}\right)+u_{j}\right),
$$

Proof. By Lemma 2.1, $\stackrel{\circ}{M}_{P}=\left(\mathbb{C}^{\times}\right)^{N} / /{ }_{\nu} K$ where $K \subset \mathbb{T}^{N}$ is a closed subgroup. By Lemma 3.3 the dual potential $\varphi_{P}^{*}$ on $\stackrel{\circ}{P}$ is given by $\varphi_{P}^{*}=\varphi^{*} \circ \iota_{\lambda}$, where $\varphi^{*}$ is the potential on the open orthant in $\left(\mathbb{R}^{N}\right)^{*}$ dual to the flat metric potential $\varphi(z)=\|z\|^{2}$ on $\left(\mathbb{C}^{\times}\right)^{N}$. By Example $4.3 \varphi^{*}=\sum e_{j} \log e_{j}$. Since $\iota_{\lambda}^{*} e_{j}=u_{j}-\lambda_{j}$,

$$
\varphi_{P}^{*}=\sum\left(u_{j}-\lambda_{j}\right) \log \left(u_{j}-\lambda_{j}\right) .
$$

By Lemmas 4.1 and 4.2 , the potential $\varphi_{P}$ is given by

$$
\varphi_{P}=\phi_{P}^{*} h
$$

where

$$
h=\sum\left(\log \left(u_{j}-\lambda_{j}\right)+1\right) u_{j}-\sum\left(u_{j}-\lambda_{j}\right) \log \left(u_{j}-\lambda_{j}\right)
$$

(see (4-1)). Therefore

$$
\varphi_{P}=\phi_{P}^{*}\left(\sum_{i=1}^{N}\left(\lambda_{j} \log \left(u_{j}-\lambda_{j}\right)+u_{j}\right)\right) .
$$

\section{Kähler potentials on the preimages of faces}

Once again let $P \subset \mathfrak{g}^{*}$ be a polyhedral set given by (2-1). Recall that in Section 2 we canonically associated to this set a Kähler quotient $M_{P}$ of $\mathbb{C}^{N}$ which carries an effective holomorphic and Hamiltonian action of the torus $G$ with a moment map $\phi_{P}: M_{P} \rightarrow \mathfrak{g}^{*}$. Let $F \subset P$ be a face. Its interior $\stackrel{\circ}{F}$ is given by

$$
\stackrel{\circ}{F}=\bigcap_{j \notin I}\left\{\eta \in \mathfrak{g}^{*} \mid\left\langle\eta, u_{j}\right\rangle-\lambda_{j}>0\right\} \cap \bigcap_{j \in I}\left\{\eta \in \mathfrak{g}^{*} \mid\left\langle\eta, u_{j}\right\rangle-\lambda_{j}=0\right\}
$$

for some nonempty subset $I$ of $\{1, \ldots, N\}$. We have seen in the proof of Lemma 2.1 that the preimage

$$
M_{\check{F}}:=\phi_{P}^{-1}(\stackrel{\circ}{F})
$$


is the Kähler quotient of $\stackrel{\circ}{V}_{I}$ by a compact abelian group $K_{I}$. Therefore there is a potential $\varphi_{F}^{*} \in C^{\infty}(\stackrel{\circ}{F})$ dual to the Kähler potential $\varphi_{F}$ on $M_{F}^{\circ}$. The goal of this section is to compute the dual potential $\varphi_{F}^{*}$ "explicitly." Lemmas 4.1 and 4.2 will then give us an analogue of (1-1) for the Kähler metric on $M_{F}^{\circ}$.

The Kähler potential $\varphi_{I}$ on $\stackrel{\circ}{V}_{I}$ for the flat metric induced from $\mathbb{C}^{N}$ is given by

$$
\varphi_{I}(z)=\sum_{j \notin I}\left|z_{j}\right|^{2} .
$$

The restriction of the moment map $\phi: \mathbb{C}^{N} \rightarrow\left(\mathbb{R}^{N}\right)^{*}$ to $\stackrel{\circ}{V}_{I}$ is a moment map for the action of the torus

$$
H_{I}:=\mathbb{T}^{N} / \mathbb{T}_{I}^{N}
$$

Note that

$$
\phi\left(\stackrel{\circ}{V}_{I}\right)=\left\{\sum_{i \notin I} a_{i} e_{i}^{*} \mid a_{i}>0\right\} .
$$

This set is an open subset in

$$
\operatorname{span}_{i \notin I}\left\{e_{j}^{*}\right\} \simeq \mathfrak{h}_{I}^{*}
$$

From now on we identify $\mathfrak{h}_{I}^{*}$ with $\operatorname{span}_{i \notin I}\left\{e_{j}^{*}\right\}$. The dual potential $\varphi_{I}^{*} \in C^{\infty}\left(\phi\left(\stackrel{\circ}{V}_{I}\right)\right)$ is easily seen to be

$$
\varphi_{I}^{*}=\sum_{j \notin I} e_{j} \log e_{j}
$$

The manifold $M_{F}^{\circ}$ is a Hamiltonian $G$ space, but the group $G$ doesn't act effectively. So we cannot yet apply Lemma 3.3 as we would like. Let $G_{I}$ denote the quotient of $G$ that does act effectively on $M_{\dot{F}}$. It is isomorphic to the quotient $H_{I} / K_{I}$. The dual of its Lie algebra $\mathfrak{g}_{I}^{*}$ is naturally embedded in $\mathfrak{g}^{*}$ :

$$
\mathfrak{g}_{I}^{*}=\left\{\eta \in \mathfrak{g}^{*} \mid\left\langle\eta, u_{i}\right\rangle=0 \text { for all } i \in I\right\} .
$$

Note also that the affine span affspan $\stackrel{\circ}{F}$ of $\stackrel{\circ}{F} \subset \mathfrak{g}^{*}$ is the translation of $\mathfrak{g}_{I}^{*}$ by an element $\eta_{0} \in \stackrel{\circ}{F}$, as it should be. Let $\gamma_{I}: \mathfrak{g}_{I}^{*} \rightarrow$ affspan $\stackrel{\circ}{F} \subset \mathfrak{g}^{*}$ denote the affine embedding. Then there exists an affine embedding $\iota_{I}: \mathfrak{g}_{I}^{*} \hookrightarrow \mathfrak{h}_{I}^{*}$ so that the diagram

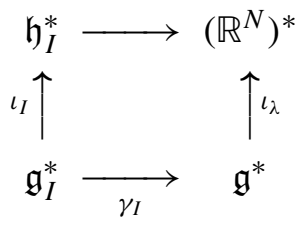

commutes. Here the top arrow identifies $\mathfrak{h}_{I}^{*}$ with $\operatorname{span}_{i \notin I}\left\{e_{i}^{*}\right\}$. Since $\gamma_{I}$ is an embedding, we may think of $\varphi_{F}^{*}$ as living on $\stackrel{\circ}{F} \subset \gamma_{I}\left(\mathfrak{g}_{I}^{*}\right)$. Therefore, by Lemma 3.3,

$$
\varphi_{F}^{*}=\left.\left(\varphi_{I}^{*} \circ \iota_{\lambda}\right)\right|_{F} ^{\circ} .
$$


Let

$$
v_{j}=\left.u_{j}\right|_{\stackrel{\circ}{F}} .
$$

These functions are affine, but not necessarily linear. Then

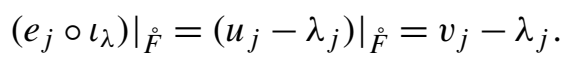

Therefore

$$
\varphi_{F}^{*}=\left.\left(\varphi_{I}^{*} \circ \iota_{\lambda}\right)\right|_{\digamma}=\sum_{j \notin I}\left(v_{j}-\lambda_{j}\right) \log \left(v_{j}-\lambda_{j}\right) .
$$

To get a nicer formula for the potential on $M_{\mathscr{F}}$ we now make a simplifying assumption, namely, that $0 \in \stackrel{\circ}{F}$. Then $v_{j}=\left.u_{j}\right|_{\mathfrak{g}_{I}^{*}}$ and, in particular, it is linear for all $j$. Hence Lemmas 4.1 and 4.2 apply, and we obtain:

Theorem 5.1. Under the simplifying assumption above, the Kähler form $\omega_{F}$ on $M_{\dot{F}}$ is given by

$$
\omega_{F}=\sqrt{-1} \partial \bar{\partial}\left(\left.\phi_{P}\right|_{M_{F}}\right) *\left(\sum_{j \notin I} \lambda_{j} \log \left(v_{j}-\lambda_{j}\right)+v_{j}\right) .
$$

Alternatively we may take the isomorphism $\gamma_{I}: \mathfrak{g}_{I}^{*} \rightarrow$ affspan $\stackrel{\circ}{F}$ explicitly into account and think of $\varphi_{F}^{*}$ as living on an open subset of $\mathfrak{g}_{I}^{*}$. Then, by Lemma 3.3,

$$
\varphi_{F}^{*}=\varphi_{I}^{*} \circ \iota_{\lambda} \circ \gamma_{I} \text {. }
$$

Since

$$
e_{i} \circ \iota_{\lambda} \circ \gamma_{I}=\left.u_{i}\right|_{\mathfrak{g}_{I}^{*}}+u_{i}\left(\eta_{0}\right)-\lambda_{i},
$$

we get

$$
\varphi_{F}^{*}=\sum_{i \notin I}\left(\left.u_{j}\right|_{\mathfrak{g}_{I}^{*}}+u_{j}\left(\eta_{0}\right)-\lambda_{j}\right) \log \left(\left.u_{j}\right|_{\mathfrak{g}_{I}^{*}}+u_{j}\left(\eta_{0}\right)-\lambda_{j}\right) .
$$

We conclude:

Theorem 5.2. The Kähler form $\omega_{F}$ on $M_{F}^{\circ}$ is given by

$$
\omega_{F}=\sqrt{-1} \partial \bar{\partial}\left(\left.\phi_{P}\right|_{M_{\bar{F}}}\right)^{*}\left(\sum_{j \notin I}\left(\left(\lambda_{j}-u_{j}\left(\eta_{0}\right)\right) \log \left(\left.u_{j}\right|_{\mathfrak{g}_{I}^{*}}+u_{j}\left(\eta_{0}\right)-\lambda_{j}\right)+\left.u_{j}\right|_{\mathfrak{g}_{I}^{*}}\right)\right) \text {. }
$$

Variations on the theme. The same technique allows us to prove a variant of (1-1). We keep the notation above. Suppose that the polyhedral set $P$ is compact. That is, suppose that $P$ is actually a polytope. Then

$$
\iota_{\lambda}(P) \subset\left\{\ell \in\left(\mathbb{R}^{N}\right)^{*} \mid\left\langle\ell, e_{j}\right\rangle \geq 0 \text { for all } j\right\}
$$

is bounded. Hence there is $R>0$ such that $\iota_{\lambda}(P)$ is contained in a scaled copy $\Delta_{R}$ of the standard simplex

$$
\Delta_{R}=\left\{\ell \in\left(\mathbb{R}^{N}\right)^{*} \mid\left\langle\ell, e_{j}\right\rangle \geq 0 \text { for all } j \text { and } \sum\left\langle\ell, e_{j}\right\rangle \leq R\right\} .
$$


Since $\Delta_{1}$ is the moment map image of $\mathbb{C P}^{N}$ under the standard action of $\mathbb{T}^{N}$, it follows that $M_{P}$ is also a symplectic quotient of $\left(\mathbb{C P}^{N}, R \omega_{F S}\right)$ by the action of the compact abelian Lie group $K$ defined earlier $\left(\omega_{F S}\right.$ denotes the Fubini-Study form; see Lemma 2.1 (3)). Since

$$
\Delta_{R}=\left\{\ell \in\left(\mathbb{R}^{N}\right)^{*} \mid\left\langle\ell, e_{j}\right\rangle \geq 0,1 \leq j \leq N,\left\langle\ell,-\sum e_{j}\right\rangle+R \geq 0\right\},
$$

it follows from (3-1) that the potential $f^{*}$ dual to the potential for $R \omega_{F S}$ on $\Delta_{R}$ is given by

$$
f^{*}=\sum e_{j} \log e_{j}+\left(R-\sum e_{j}\right) \log \left(R-\sum e_{j}\right) .
$$

Consequently the potential $f_{v}^{*}$ dual to the potential on the quotient $\left(\mathbb{C P}^{N} / /{ }_{v} K, \omega_{P}\right)$ is

$$
f_{v}^{*}=\sum\left(u_{j}-\lambda_{j}\right) \log \left(u_{j}-\lambda_{j}\right)+\left(R-\sum\left(u_{j}-\lambda_{j}\right)\right) \log \left(R-\sum\left(u_{j}-\lambda_{j}\right)\right) .
$$

By Lemma 4.1 the reduced Kähler form $\omega_{P}$ is

$$
\omega_{P}=\sqrt{-1} \partial \bar{\partial} \phi^{*} h
$$

where

$$
h(\eta)=\left\langle\eta,\left(d f_{v}\right)_{\eta}\right\rangle-f_{v}(\eta) .
$$

A computation similar to the ones in the previous sections gives

$$
h=\sum \lambda_{j} \log \left(u_{j}-\lambda_{j}\right)-\left(R+\sum \lambda_{j}\right) \log \left(R-\sum\left(u_{j}-\lambda_{j}\right)\right) .
$$

We have proved the following theorem.

Theorem 5.3. Let $G$ be a torus, $P \subset \mathfrak{g}^{*}$ the polyhedral set defined by (2-1) which happens to be compact, $M_{P}=\left(\mathbb{C P}{ }^{N}, R \omega_{F S}\right) / /{ }_{\nu} K$ the Kähler $G$-space with moment map $\phi_{P}: M_{P} \rightarrow \mathfrak{g}^{*}$ constructed in Lemma 2.1 (3). Then the Kähler form $\omega_{P}$ on $\stackrel{\circ}{M}_{P}:=\phi_{P}^{-1}(\stackrel{\circ}{P})$ is given by

$$
\omega_{P}=\sqrt{-1} \partial \bar{\partial} \phi_{P}^{*}\left(\sum \lambda_{j} \log \left(u_{j}-\lambda_{j}\right)-\left(R+\sum \lambda_{j}\right) \log \left(R-\sum\left(u_{j}-\lambda_{j}\right)\right)\right) .
$$

\section{Acknowledgments}

We thank Peter Heinzner for patiently answering our queries and the referee for valuable comments.

\section{References}

[Abreu 2001] M. Abreu, "Kähler metrics on toric orbifolds", J. Differential Geom. 58:1 (2001), 151-187. MR 2003b:53046 Zbl 1035.53055

[Burns and Guillemin 2004] D. Burns and V. Guillemin, "Potential functions and actions of tori on Kähler manifolds”, Comm. Anal. Geom. 12:1-2 (2004), 281-303. MR 2005e:53133 Zbl 1073. 53113 
[Calderbank et al. 2003] D. M. J. Calderbank, L. David, and P. Gauduchon, "The Guillemin formula and Kähler metrics on toric symplectic manifolds", J. Symplectic Geom. 1:4 (2003), 767-784. MR 2005a:53145 Zbl 02199608

[Guillemin 1994] V. Guillemin, "Kaehler structures on toric varieties”, J. Differential Geom. 40:2 (1994), 285-309. MR 95h:32029 Zbl 0813.53042

[Guillemin and Sternberg 1982] V. Guillemin and S. Sternberg, "Geometric quantization and multiplicities of group representations", Invent. Math. 67:3 (1982), 515-538. MR 83m:58040 Zbl 0503. 58018

[Heinzner and Huckleberry 1996] P. Heinzner and A. Huckleberry, "Kählerian potentials and convexity properties of the moment map", Invent. Math. 126:1 (1996), 65-84. MR 98e:58075 Zbl 0855.58025

[Heinzner and Loose 1994] P. Heinzner and F. Loose, "Reduction of complex Hamiltonian Gspaces”, Geom. Funct. Anal. 4:3 (1994), 288-297. MR 95j:58050 Zbl 0816.53018

[Heinzner et al. 1994] P. Heinzner, A. T. Huckleberry, and F. Loose, "Kählerian extensions of the symplectic reduction”, J. Reine Angew. Math. 455 (1994), 123-140. MR 95k:58061 Zbl 0803. 53042

[Martelli and Sparks 2004] D. Martelli and J. Sparks, “Toric geometry, Sasaki-Einstein manifolds and a new infinite class of AdS/CFT duals", preprint, 2004. arXiv hep-th/0411238

Received September 10, 2007. Revised February 29, 2008.

DAN BURNS

DEPARTMENT OF MATHEMATiCS

UNIVERSITY OF MICHIGAN

ANN ARBOR, MI 48109-1043

UNITED STATES

dburns@umich.edu

ViCTOR GUILLEMIN

DEPARTMENT OF MATHEMATICS

MAssachussetTs Institute of TECHNOLOGY

CAMBRIDGE, MA 02139-4307

UNITED STATES

vwg@math.mit.edu

EUGENE LERMAN

DEPARTMENT OF MATHEMATICS

UNIVERSITY OF ILLINOIS

URBANA, IL 61801

UNITED STATES

lerman@math.uiuc.edu 